Check for updates

Cite this: RSC Adv., 2021, 11, 2221

Received 22nd January 2020

Accepted 2nd December 2020

DOI: $10.1039 / \mathrm{d} 0 \mathrm{ra00686f}$

rsc.li/rsc-advances

\section{A mild one-pot synthesis of 2-iminothiazolines from thioureas and 1-bromo-1-nitroalkenes $\dagger$}

\author{
Yuan Xu, ab Xin Ge, ${ }^{b}$ Yuhan Zhang, ${ }^{b}$ Hongbin Zhang (D) and Xue-Wei Liu (D)*b \\ A mild method to access functionalized 2-iminothiazolines in a facile and efficient manner has been \\ developed. The reaction started from 1,3-disubstituted thioureas and 1-bromo-1-nitroalkenes in the \\ presence of triethylamine in THF and proceeded smoothly in air to afford 2-iminothiazoline derivatives in \\ moderate to good yields.
}

Functionalized 2-iminothiazoline has been an important building block in organic chemistry. ${ }^{1}$ Its derivatives show significant pharmacological activities such as bactericidal and fungicidal activity. ${ }^{2}$ In addition, they are found in drug applications for treatment of allergies, hypertension, inflammations, bacterial and HIV infections. ${ }^{3}$ Pifithrin (Pft- $\alpha$ ) (Fig. 1), isolated by screening of chemical libraries using 2-iminothiazoline skeleton, is a lead compound for p53 inactivation and has received increasing attention due to its possible applications in therapy of Alzheimer's disease, Parkinson's disease, stroke and other pathologies related to various signalling pathways. ${ }^{4}$ Hence, its utility and applicability are widely recognized in organic and biological areas.

The classical synthesis of 2-aminothiazole moiety involves the Hantzsch condensation reaction of thioureas and $\alpha$-haloketones. ${ }^{5}$ Birkinshaw et al. reported the synthesis of $N$-alkylated imino-thiazolines by replacing thioureas with mono- $N$ substituted thioureas. ${ }^{6}$ Also, several alternative strategies have been devised, which include synthesis of highly functionalized
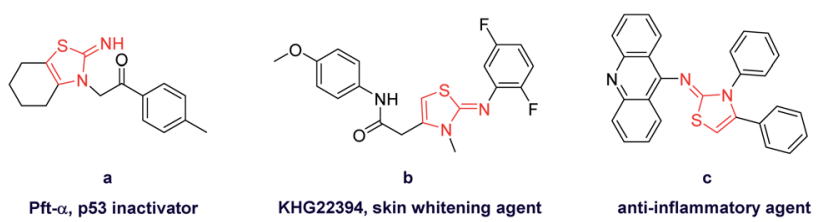

Fig. 1 Pharmacologically important molecules consisting of 2-iminothiazoline core structure. (a) p53 inactivator; (b) skin whitening agent; (c) anti-inflammatory agent.

${ }^{a}$ Key Laboratory of Medicinal Chemistry for Natural Resource, Ministry of Education and Yunnan Province, School of Chemical Science and Technology, Yunnan University, Kunming 650091, China

${ }^{b}$ Division of Chemistry and Biological Chemistry, School of Physical and Mathematical Sciences, Nanyang Technological University, 637371, Singapore.E-mail: xuewei@ntu. edu.sg

$\dagger$ Electronic supplementary information (ESI) available: NMR spectra of products. See DOI: $10.1039 /$ d0ra00686f thiazoles and 2 -iminothiazolines by replacing $\alpha$-haloketone with 2,2-dicyano-3,3-bis(trifluoromethyl)oxirane ${ }^{7}$ and 2-chlorooxirane,,$^{8}$ treatment of $\alpha$-bromoketimines with potassium thiocyanate, ${ }^{9}$ reaction of $N$-monoalkylated thioureas with 3 bromomethyl-2-cyanocinnamonitrile, ${ }^{\mathbf{1 0}}$ cycloadditions followed by elimination of 5-imino-1,2,4-thiazolidin-3-ones with enamines and ester enolate, ${ }^{11}$ ring transformation of 1-

Table 1 Reaction of $\beta$-bromo- $\beta$-nitrostyrene 1 a with 1,3-diphenylthiourea 2a under different conditions

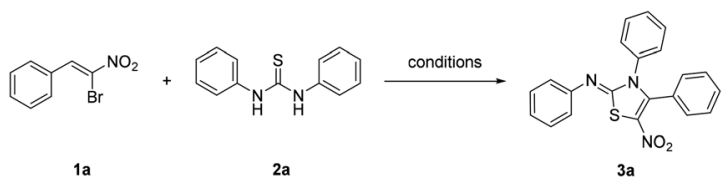

\begin{tabular}{llllll}
\hline & & & & \\
Entry $^{a}$ & Base & Solvent & $\left({ }^{\circ} \mathrm{C}\right)$ & Time (h) & Yield (\%) \\
\hline 1 & & & & & \\
$2^{b}$ & $\mathrm{~K}_{2} \mathrm{CO}_{3}$ & $\mathrm{THF}$ & $\mathrm{rt}$ & 24 & 62 \\
3 & $\mathrm{~K}_{2} \mathrm{CO}_{3}$ & $\mathrm{THF}$ & 70 & 10 & 60 \\
$4^{b}$ & $\mathrm{Et}_{3} \mathrm{~N}$ & $\mathrm{THF}$ & $\mathrm{rt}$ & 24 & 72 \\
5 & $\mathrm{Et}_{3} \mathrm{~N}$ & $\mathrm{THF}$ & 70 & 10 & 65 \\
$6^{b}$ & $\mathrm{DBU}$ & $\mathrm{THF}$ & $\mathrm{rt}$ & 24 & 63 \\
7 & $\mathrm{DBU}^{b}$ & $\mathrm{THF}$ & 70 & 10 & 58 \\
$8^{b}$ & $\mathrm{KHCO}_{3}$ & $\mathrm{THF}$ & $\mathrm{rt}$ & 24 & 55 \\
9 & $\mathrm{KHCO}_{3}$ & $\mathrm{THF}$ & 70 & 10 & 60 \\
10 & $\mathrm{DIPEA}^{b}$ & $\mathrm{THF}$ & $\mathrm{rt}$ & 24 & 68 \\
$11^{c}$ & $\mathrm{None}$ & $\mathrm{THF}$ & $\mathrm{rt}$ & 24 & 29 \\
$12^{d}$ & $\mathrm{Et}_{3} \mathrm{~N}$ & $\mathrm{THF}$ & $\mathrm{rt}$ & 24 & 64 \\
$13^{e}$ & $\mathrm{Et}_{3} \mathrm{~N}$ & $\mathrm{THF}$ & $\mathrm{rt}$ & 24 & 58 \\
14 & $\mathrm{Et}_{3} \mathrm{~N}$ & $\mathrm{THF}$ & $\mathrm{rt}$ & 24 & 17 \\
15 & $\mathrm{Et}_{3} \mathrm{~N}$ & $\mathrm{CH}{ }_{2} \mathrm{Cl}{ }_{2}$ & $\mathrm{rt}$ & 24 & 45 \\
$16^{b}$ & $\mathrm{Et}_{3} \mathrm{~N}$ & $\mathrm{Toluene}$ & $\mathrm{rt}$ & 24 & 42 \\
& $\mathrm{Et}_{3} \mathrm{~N}$ & Toluene & 110 & 5 & 40
\end{tabular}

${ }^{a}$ Reactions were performed with $\beta$-bromo- $\beta$-nitrostyrene $1 \mathrm{a}(0.10 \mathrm{mmol})$ and 1,3-diphenylthiourea $2 \mathrm{a}(0.11 \mathrm{mmol})$ with base $(0.02 \mathrm{mmol})$ in the indicated solvent $(2.0 \mathrm{~mL})$ under atmospheric air. ${ }^{b} \beta$-Bromo- $\beta$ nitrostyrene was completely consumed. ${ }^{c}$ Reaction was carried out with $0.04 \mathrm{mmol}$ of base. ${ }^{d}$ Reaction was carried out with $0.01 \mathrm{mmol}$ of base. ${ }^{e}$ Reaction was carried out with $0.1 \mathrm{mmol}$ of base. 


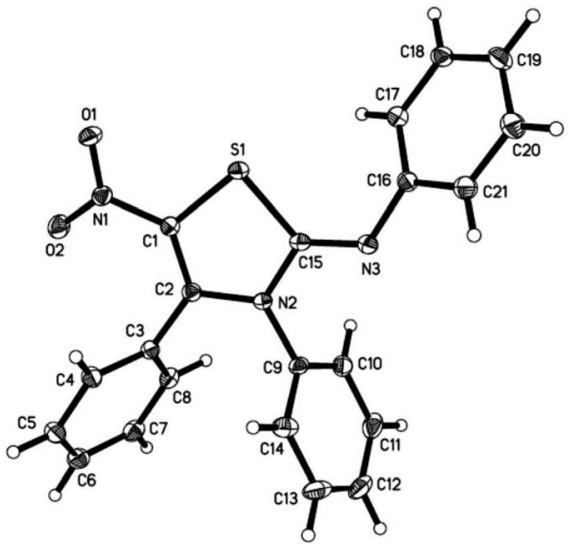

Fig. 2 X-ray crystallography of compound 3a.

Table 2 Reaction of various 1-bromo-1-nitroalkenes 1a-1m with 1,3diphenylthiourea $2^{a}$

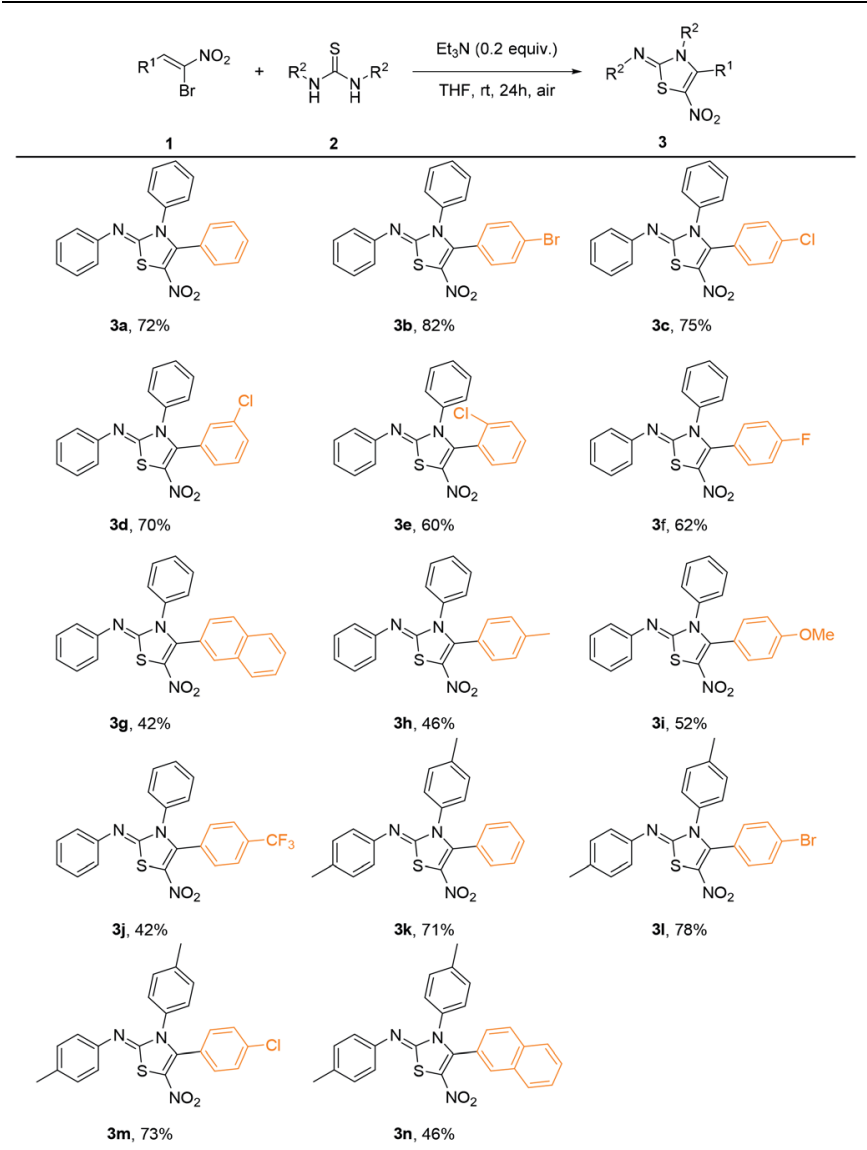

${ }^{a}$ Reactions were performed with 1-bromo-1-nitroalkenes 1 a-1n $(0.10$ $\mathrm{mmol})$ and 1,3-diarylthioureas $2(0.11 \mathrm{mmol})$ with $\mathrm{Et}_{3} \mathrm{~N}(0.02 \mathrm{mmol})$ in THF $(2.0 \mathrm{~mL})$ at room temperature in the air for 24 hours.

arylmethyl-2-(thiocyanomethyl)aziridines in the presence of $\mathrm{TiCl}_{4}$ and acyl chloride, ${ }^{12}$ reaction of $\mathrm{N}$-propargylaniline with acyl isothiocyanates. ${ }^{13}$ Less general approaches towards the synthesis of these compounds involve the reaction of ketone either with $\mathrm{N}$-alkyl rhodanamine or bisbenzyl formamidine disulfide $^{\mathbf{1 4}}$ or the reaction of $\alpha$-chloroketones with thiosemicarbazide in an acidic medium, ${ }^{\mathbf{1 5}}$ condensation of $\alpha$-haloketones with $N$-benzoyl- $N^{\prime}$-arylthioureas or $N, N^{\prime}$-disubstituted thioureas. ${ }^{16}$

Although some of the methods used for preparing 2-iminothiazolines are convenient and effective, most procedures reported in literatures require arduous preparation of precursor substrates or harsh reaction conditions. Till now, only a few procedures on the one-pot synthesis of 2-iminothiazoline from $N, N^{\prime}$-dialkylthiourea and in situ generated $\alpha$-bromoketones have been reported. ${ }^{17}$ Herein, we reported a novel and efficient methodology for the synthesis of 2-imino-5-nitrothiazolines using 1,3-diarylthioureas and 1-bromo-1-nitroalkenes as starting materials.

The $\beta$-bromo- $\beta$-nitrostyrenes, with $\beta$-disubstituted styrene structure, showed versatile reactivity as a trifunctional synthon. Previous literatures showed their activity as Michael acceptors and $[3+2]$ and $[4+2]$ cycloaddition partners. ${ }^{18}$ In our preliminary experiments, the reaction of 1,3-diphenylthiourea and $\beta$ bromo- $\beta$-nitrostyrene 1a was studied, while the latter one could easily be prepared according to the reported procedure. ${ }^{18 e} \mathrm{We}$ found that when these two reactants were treated with base such as $\mathrm{K}_{2} \mathrm{CO}_{3}$ in THF at room temperature under atmospheric air, a red crystalline product was obtained (Table 1, entry 1).

This structure was later confirmed by single crystal X-ray analysis as shown in Fig. 2. When the reaction temperature was increased to $70{ }^{\circ} \mathrm{C}$, the reaction was completed in shorter time. However, the yield was lower due to an increase of side products (Table 1 , entry 2).

Several other bases were tested such as $\mathrm{K}_{2} \mathrm{CO}_{3}, \mathrm{Et}_{3} \mathrm{~N}, \mathrm{DBU}$, $\mathrm{KHCO}_{3}$, DIPEA, and $\mathrm{Et}_{3} \mathrm{~N}$ was found to give the best results (Table 1, entries 1-9). When the reaction was conducted without base, most starting material remained, and very less desired product was obtained (Table 1, entry 10). Further examination was focussed on base loadings, providing the best results at 0.2 equiv. of base (Table 1, entries 11-13). Higher loading of base resulted in more side products rather than desired product while less or no loading of base were not enough to accelerate the reaction. These results suggest that suitable basicity and loading of employed base are critical for the competition between desired and side reactions. Different solvents were screened, and THF was identified to be the optimal solvent (Table 1, entries 14-16). When the reaction was conducted in an inert atmosphere, almost no desired products were formed. From the optimization results, we concluded that 0.2 equiv. of $\mathrm{Et}_{3} \mathrm{~N}$ in THF at room temperature in the air presented the best set of condition for this reaction.

With the optimized conditions in hand, we investigated the scope of this reaction, and the results were shown in Table 2. In general, $\beta$-bromo- $\beta$-nitrostyrenes bearing halogen substituents on the phenyl ring give moderate to good yields (3b-3f) while stronger electron-donating and electron-withdrawing groups afford the products in relatively lower yields (3h-3j). While different types of thioureas including 1,3-diaryl, 1-aryl-3-alkyl and 1,3-dialkyl substituted ones were employed, only symmetrical 1,3-diarylthiourea could afford desired products. As an 


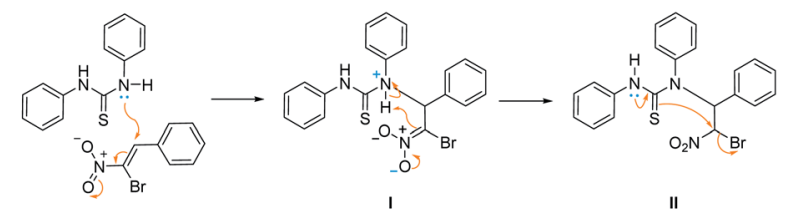

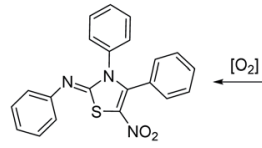

3a

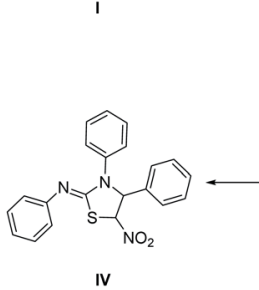

IV

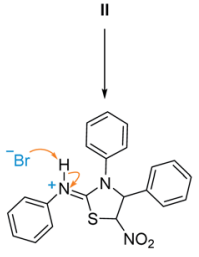

III
Scheme 1 Plausible reaction pathway.

example, 1,3-ditolylthiourea could undergo the transformation smoothly under the optimal reaction condition, affording the desired products in moderate to good yields (3k-3n).

A plausible mechanism for this reaction has been proposed as shown in Scheme 1. Initially, a typical Michael addition happens with the $\beta$-bromo- $\beta$-nitrostyrene $\mathbf{1 a},{ }^{19}$ which is initiated by the attack of lone pair of nitrogen atom in 1,3-diphenylthiourea 2a, affording intermediate II. The successive tautomerism of thiourea structure and nucleophilic substitution in intermediate II generates five-membered ring intermediate III. Deprotonation of intermediate III by preceding bromide ion affords intermediate IV which subsequently undergoes aromatization with aid of atmospheric oxygen, ${ }^{20}$ yielding final product 3a.

\section{Conclusions}

In summary, we have developed a facile and mild synthesis to functionalized 2-imino-5-nitrothiazoline by reacting 1-bromo-1nitroalkenes with 1,3-diarylthioureas in a mild basic condition. Although this method is limited to symmetrical thioureas, it provides a new approach for the synthesis of diverse 2-iminothiazolines, in which 1-bromo-1-nitroalkenes were used as a trifunctional synthon.

\section{Experimental}

${ }^{1} \mathrm{H}$ and ${ }^{13} \mathrm{C}$ nuclear magnetic resonance (NMR) spectra were recorded on Bruker AV400 (400 MHz) or AV500 (500 MHz) NMR spectrometer. ${ }^{1} \mathrm{H}$ and ${ }^{13} \mathrm{C}$ NMR spectra are reported in parts per million (ppm) downfield from an internal standard, tetramethylsilane (0 ppm for $\left.{ }^{1} \mathrm{H}\right)$ and $\mathrm{CHCl}_{3}\left(7.26 \mathrm{ppm}\right.$ for ${ }^{1} \mathrm{H}$ and $77.0 \mathrm{ppm}$ for ${ }^{13} \mathrm{C}$ ). High-resolution mass spectra (HRMS) were obtained on a Finnigan/MAT LCQ quadrupole ion trap mass spectrometer, coupled with the TSP4000 HPLC system and the Crystal $310 \mathrm{CE}$ system. Accurate masses are reported for the molecular ion $[\mathrm{M}+\mathrm{H}]^{+}$or a suitable fragment ion. X-ray crystallographic data was collected by using a Bruker X8 Apex diffractometer with Mo $\mathrm{K} \alpha$ radiation (graphite monochromator). All reagents were purchased from commercial suppliers and used without further purification except where noted otherwise. All reactions were conducted under an atmosphere of air, unless otherwise indicated. Analytical thin-layer chromatography (TLC) was performed on Merck 60 F254 silica gel plates. Flash chromatography was performed on silica gel 60 (0.010-0.063 mm, Merck). 1-Bromo-1-nitroalkenes 1a-1m were prepared from the standard literature procedures. ${ }^{18 e}$ 1,3-Diarylthioureas were obtained from commercial suppliers and used without further purification.

\section{General procedure for synthesis of compound 3}

To a well-stirred solution of $\beta$-bromo- $\beta$-nitrostyrene 1 (0.1 mmol, 1.0 equiv., prepared as a mixture of $Z$ - and $E$ isomers), 1,3-diarylthiourea 2 ( $0.11 \mathrm{mmol}, 1.1$ equiv.) in THF $(2.0 \mathrm{~mL})$ was added triethylamine $(2.0 \mathrm{mg}, 0.02 \mathrm{mmol}, 0.2$ equiv.). The reaction mixture was stirred at room temperature in the air for $24 \mathrm{~h}$ (TLC monitored). The resulting mixture was concentrated under reduced pressure to give the crude residue. The crude residue was then purified by flash column chromatography on silica gel (10\% EtOAc in hexanes) to afford final product.

\section{Characterization of compounds $3 \mathbf{a}-3 \mathbf{n}$}

3a: (red crystal, 72\%). ${ }^{1} \mathrm{H}$ NMR (400 $\left.\mathrm{MHz}, \mathrm{CDCl}_{3}\right): \delta$ 7.36-7.27 $(\mathrm{m}, 8 \mathrm{H}), 7.24-7.17(\mathrm{~m}, 4 \mathrm{H}), 7.13(\mathrm{t}, J=7.2 \mathrm{~Hz}, 1 \mathrm{H}), 7.00(\mathrm{dd}, J=$ 8.0, $1.2 \mathrm{~Hz}, 2 \mathrm{H}) ;{ }^{13} \mathrm{C} \mathrm{NMR}\left(100 \mathrm{MHz}, \mathrm{CDCl}_{3}\right) \delta$ 153.9, 149.6, 146.5, 135.5, 130.2, 129.7, 129.4, 129.2, 129.02, 128.99, 128.3, $127.5,124.7,120.6$. HRMS (ESI) $m / z$ calcd for $\mathrm{C}_{21} \mathrm{H}_{16} \mathrm{~N}_{3} \mathrm{O}_{2} \mathrm{~S}[\mathrm{M}+$ $\mathrm{H}]^{+}: 374.0963$, found 374.0952 .

3b: (red crystal, 82\%). ${ }^{1} \mathrm{H}$ NMR (400 $\left.\mathrm{MHz}, \mathrm{CDCl}_{3}\right): \delta 7.43(\mathrm{~d}, J$ $=8.4 \mathrm{~Hz}, 2 \mathrm{H}), 7.38-7.30(\mathrm{~m}, 5 \mathrm{H}), 7.19-7.09(\mathrm{~m}, 5 \mathrm{H}), 6.99(\mathrm{~d}, J=$ $7.6 \mathrm{~Hz}, 2 \mathrm{H}) ;{ }^{13} \mathrm{C} \mathrm{NMR}\left(100 \mathrm{MHz}, \mathrm{CDCl}_{3}\right) \delta 153.8,149.7,145.3$, 135.4 , 131.9, 131.2, 129.9, 129.6, 129.4, 129.1, 126.4, 125.1, 125.0, 120.7. HRMS (ESI) $m / z$ calcd for $\mathrm{C}_{21} \mathrm{H}_{15}{ }^{79} \mathrm{BrN}_{3} \mathrm{O}_{2} \mathrm{~S}[\mathrm{M}+$ $\mathrm{H}]^{+}$: 452.0068, found 452.0070; $\mathrm{C}_{21} \mathrm{H}_{15}{ }^{81} \mathrm{BrN}_{3} \mathrm{O}_{2} \mathrm{~S}[\mathrm{M}+\mathrm{H}]^{+}$: 454.0048, found 454.0052 .

3c: (yellow crystal, 75\%). ${ }^{1} \mathrm{H}$ NMR $\left(400 \mathrm{MHz}, \mathrm{CDCl}_{3}\right): \delta 7.38-$ $7.26(\mathrm{~m}, 7 \mathrm{H}), 7.19-7.11(\mathrm{~m}, 5 \mathrm{H}), 6.99(\mathrm{~d}, J=8.4 \mathrm{~Hz}, 2 \mathrm{H}) ;{ }^{13} \mathrm{C}$ NMR (100 MHz, $\left.\mathrm{CDCl}_{3}\right) \delta 153.8,149.7,145.3,136.8,135.5,131.0$, 129.9, 129.6, 129.4, 129.1 128.9, 126.0, 125.0, 120.7. HRMS (ESI) $m / z$ calcd for $\mathrm{C}_{21} \mathrm{H}_{15}{ }^{35} \mathrm{ClN}_{3} \mathrm{O}_{2} \mathrm{~S}[\mathrm{M}+\mathrm{H}]^{+}$: 408.0574, found 408.0571; $\mathrm{C}_{21} \mathrm{H}_{15}{ }^{37} \mathrm{ClN}_{3} \mathrm{O}_{2} \mathrm{~S}[\mathrm{M}+\mathrm{H}]^{+}$: 410.0544, found 410.0540.

3d: (yellow crystal, 70\%). ${ }^{1} \mathrm{H}$ NMR (400 MHz, $\left.\mathrm{CDCl}_{3}\right): \delta 7.38-$ 7.32 (m, 6H), 7.30-7.24 (m, 2H), 7.22 (d, $J=10.8 \mathrm{~Hz}, 2 \mathrm{H}), 7.18-$ $7.10(\mathrm{~m}, 2 \mathrm{H}), 6.99(\mathrm{~d}, J=7.6 \mathrm{~Hz}, 2 \mathrm{H}) ;{ }^{13} \mathrm{C} \mathrm{NMR}\left(100 \mathrm{MHz}, \mathrm{CDCl}_{3}\right)$ $\delta 153.7,149.6,144.7,135.3,134.5,130.6,129.9,129.8,129.6$, 129.53, 129.47, 129.3, 129.1, 127.7, 125.0, 120.7. HRMS (ESI) $\mathrm{m} / \mathrm{z}$ calcd for $\mathrm{C}_{21} \mathrm{H}_{15}{ }^{35} \mathrm{ClN}_{3} \mathrm{O}_{2} \mathrm{~S}[\mathrm{M}+\mathrm{H}]^{+}$: 408.0574, found 408.0576; $\mathrm{C}_{21} \mathrm{H}_{15}{ }^{37} \mathrm{ClN}_{3} \mathrm{O}_{2} \mathrm{~S}[\mathrm{M}+\mathrm{H}]^{+}: 410.0544$, found 410.0541 .

3e: (yellow solid, 60\%). ${ }^{1} \mathrm{H}$ NMR (400 $\left.\mathrm{MHz}, \mathrm{CDCl}_{3}\right): \delta 7.39-$ 7.29 (m, 9H), 7.21-7.13 (m, 3H), 7.02 (d, $J=7.2 \mathrm{~Hz}, 2 \mathrm{H}) ;{ }^{13} \mathrm{C}$ NMR $\left(100 \mathrm{MHz}, \mathrm{CDCl}_{3}\right) \delta 153.8,149.7,143.4,135.2,133.7,131.7$, 130.5, 129.9, 129.8, 129.6, 129.4, 127.7, 127.0, 125.0, 120.8. HRMS (ESI) $m / z$ calcd for $\mathrm{C}_{21} \mathrm{H}_{15}{ }^{35} \mathrm{ClN}_{3} \mathrm{O}_{2} \mathrm{~S}[\mathrm{M}+\mathrm{H}]^{+}:$408.0574, found 408.0571; $\mathrm{C}_{21} \mathrm{H}_{15}{ }^{37} \mathrm{ClN}_{3} \mathrm{O}_{2} \mathrm{~S}[\mathrm{M}+\mathrm{H}]^{+}$: 410.0544, found 410.0540 . 
3f: (yellow solid, 62\%). ${ }^{1} \mathrm{H}$ NMR (400 $\left.\mathrm{MHz}, \mathrm{CDCl}_{3}\right): \delta 7.34-$ 7.27 (m, 5H), 7.25-7.11 (m, 5H), 7.02-6.97 (m, 4H); ${ }^{13} \mathrm{C}$ NMR $\left(100 \mathrm{MHz}, \mathrm{CDCl}_{3}\right) \delta 164.9,162.4,153.8,149.7,145.6,135.6$, 132.0, 131.9, 129.9, 129.5, 129.4, 129.1, 125.0, 123.50, 123.46, $120.8,116.1,115.8$. HRMS (ESI) $\mathrm{m} / z$ calcd for $\mathrm{C}_{21} \mathrm{H}_{15} \mathrm{FN}_{3} \mathrm{O}_{2} \mathrm{~S}[\mathrm{M}$ $+\mathrm{H}]^{+}: 392.0869$, found 392.0852 .

3g: (yellow solid, $42 \%) .{ }^{1} \mathrm{H}$ NMR $\left(400 \mathrm{MHz}, \mathrm{CDCl}_{3}\right): \delta 7.77-$ $7.73(\mathrm{~m}, 4 \mathrm{H}), 7.54-7.48(\mathrm{~m}, 2 \mathrm{H}), 7.39-7.35(\mathrm{~m}, 2 \mathrm{H}), 7.28-7.22$ $(\mathrm{m}, 5 \mathrm{H}), 7.21-7.12(\mathrm{~m}, 2 \mathrm{H}), 7.02(\mathrm{~d}, J=8.4 \mathrm{~Hz}, 2 \mathrm{H}) ;{ }^{13} \mathrm{C}$ NMR $\left(100 \mathrm{MHz}, \mathrm{CDCl}_{3}\right) \delta 154.1,149.8,146.7,135.7,133.7,132.5$, $130.3,129.9,129.4,129.2,129.1,128.6,128.3,128.0,127.9$, 126.9, 125.7, 124.9, 124.8, 120.8. HRMS (ESI) $\mathrm{m} / \mathrm{z}$ calcd for $\mathrm{C}_{25} \mathrm{H}_{18} \mathrm{~N}_{3} \mathrm{O}_{2} \mathrm{~S}[\mathrm{M}+\mathrm{H}]^{+}:$: 424.1120, found 424.1125.

3h: (yellow solid, $46 \%) .{ }^{1} \mathrm{H}$ NMR $\left(400 \mathrm{MHz}, \mathrm{CDCl}_{3}\right) \delta 7.40-$ $7.23(\mathrm{~m}, 5 \mathrm{H}), 7.22-7.16(\mathrm{~m}, 2 \mathrm{H}), 7.15-7.06(\mathrm{~m}, 5 \mathrm{H}), 6.99(\mathrm{dd}, J=$ $7.8,1.2 \mathrm{~Hz}, 2 \mathrm{H}), 2.30(\mathrm{~s}, 3 \mathrm{H}) ;{ }^{13} \mathrm{C}$ NMR $\left(100 \mathrm{MHz}, \mathrm{CDCl}_{3}\right) \delta 154.2$, 149.9, 147.1, 140.8, 135.8, 129.9, 129.5, 129.3, 129.21, 129.18, 129.1, 124.9, 124.5, 120.8, 21.6. HRMS (ESI) $\mathrm{m} / \mathrm{z}$ calcd for $\mathrm{C}_{22} \mathrm{H}_{18} \mathrm{~N}_{3} \mathrm{O}_{2} \mathrm{~S}[\mathrm{M}+\mathrm{H}]^{+}: 388.1120$, found 388.1119.

3i: (yellow solid, 56\%). ${ }^{1} \mathrm{H}$ NMR $\left(400 \mathrm{MHz}, \mathrm{CDCl}_{3}\right): \delta 7.36-$ $6.99(\mathrm{~m}, 8 \mathrm{H}), 6.85-6.78(\mathrm{~m}, 6 \mathrm{H}), 3.81(\mathrm{~s}, 3 \mathrm{H}) ;{ }^{13} \mathrm{C} \mathrm{NMR}(100 \mathrm{MHz}$, $\left.\mathrm{CDCl}_{3}\right) \delta 161.1,154.1,149.8,147.0,135.9,131.4,129.8,129.4$, 129.2, 129.1, 124.8, 120.8, 119.1, 113.9, 55.4. HRMS (ESI) $\mathrm{m} / \mathrm{z}$ calcd for $\mathrm{C}_{22} \mathrm{H}_{18} \mathrm{~N}_{3} \mathrm{O}_{3} \mathrm{~S}[\mathrm{M}+\mathrm{H}]^{+}:$404.1069, found 404.1054.

3j: (yellow solid, $42 \%) .{ }^{1} \mathrm{H}$ NMR $\left(500 \mathrm{MHz}, \mathrm{CDCl}_{3}\right) \delta 7.56(\mathrm{~d}, J$ $=8.1 \mathrm{~Hz}, 2 \mathrm{H}), 7.41-7.27(\mathrm{~m}, 7 \mathrm{H}), 7.21-7.11(\mathrm{~m}, 3 \mathrm{H}), 6.99(\mathrm{dd}, J=$ 8.6, $1.1 \mathrm{~Hz}, 2 \mathrm{H}) ;{ }^{13} \mathrm{C} \mathrm{NMR}\left(125 \mathrm{MHz}, \mathrm{CDCl}_{3}\right) \delta 153.7,149.6$, 144.7, 135.2, 132.4, 132.1, 131.4, 130.2, 129.9, 129.60, 129.61, 129.0, 125.6, 125.53, 125.50, 125.47, 125.1, 124.6, 122.5, 120.7 . HRMS (ESI) $\mathrm{m} / z$ calcd for $\mathrm{C}_{22} \mathrm{H}_{15} \mathrm{~F}_{3} \mathrm{~N}_{3} \mathrm{O}_{2} \mathrm{~S}[\mathrm{M}+\mathrm{H}]^{+}: 442.0837$, found 442.0839 .

3k: (red solid, $71 \%$ ). ${ }^{1} \mathrm{H}$ NMR (500 MHz, $\left.\mathrm{CDCl}_{3}\right) \delta$ 7.37-7.25 (m, 3H), 7.25-7.20 (m, 2H), $7.16(\mathrm{~d}, J=7.9 \mathrm{~Hz}, 2 \mathrm{H}), 7.11-7.04$ $(\mathrm{m}, 4 \mathrm{H}), 6.90(\mathrm{~d}, J=7.9 \mathrm{~Hz}, 2 \mathrm{H}), 2.34(\mathrm{~s}, 3 \mathrm{H}), 2.27(\mathrm{~s}, 3 \mathrm{H}) ;{ }^{13} \mathrm{C}$ NMR $\left(125 \mathrm{MHz}, \mathrm{CDCl}_{3}\right) \delta 154.2,147.4,147.0,139.2,134.4,133.0$, 130.4, 130.3, 130.0, 129.5, 128.8, 128.4, 127.8, 120.6, 21.3, 21.1. HRMS (ESI) $m / z$ calcd for $\mathrm{C}_{23} \mathrm{H}_{20} \mathrm{~N}_{3} \mathrm{O}_{2} \mathrm{~S}[\mathrm{M}+\mathrm{H}]^{+}$: 402.1276, found 402.1280 .

31: (red solid, $78 \%) .{ }^{1} \mathrm{H}$ NMR $\left(400 \mathrm{MHz}, \mathrm{CDCl}_{3}\right) \delta 7.45-7.39$ $(\mathrm{m}, 2 \mathrm{H}), 7.17-7.06(\mathrm{~m}, 6 \mathrm{H}), 7.06-7.00(\mathrm{~m}, 2 \mathrm{H}), 6.90-6.84(\mathrm{~m}$, 2H), 2.32 (s, 3H), 2.29 (s, 3H); ${ }^{13} \mathrm{C}$ NMR (100 MHz, $\left.\mathrm{CDCl}_{3}\right)$ $\delta 153.8,147.3,145.6,139.5,134.5,131.8,131.1,130.4,130.2$, $128.7,126.6,125.0,120.5,21.4,21.1$. HRMS (ESI) $\mathrm{m} / \mathrm{z}$ calcd for $\mathrm{C}_{23} \mathrm{H}_{19}{ }^{79} \mathrm{BrN}_{3} \mathrm{O}_{2} \mathrm{~S}[\mathrm{M}+\mathrm{H}]^{+}:$480.0381, found 480.0378; HRMS (ESI) $m / z$ calcd for $\mathrm{C}_{23} \mathrm{H}_{19}{ }^{81} \mathrm{BrN}_{3} \mathrm{O}_{2} \mathrm{~S}[\mathrm{M}+\mathrm{H}]^{+}: 482.0361$, found 482.0359.

3m: (yellow solid, 73\%). ${ }^{1} \mathrm{H}$ NMR $\left(400 \mathrm{MHz}, \mathrm{CDCl}_{3}\right) \delta 7.28-$ $7.23(\mathrm{~m}, 2 \mathrm{H}), 7.18-7.12(\mathrm{~m}, 4 \mathrm{H}), 7.09(\mathrm{~d}, J=8.3 \mathrm{~Hz}, 2 \mathrm{H}), 7.05-$ $7.00(\mathrm{~m}, 2 \mathrm{H}), 6.89-6.84(\mathrm{~m}, 2 \mathrm{H}), 2.32(\mathrm{~s}, 3 \mathrm{H}), 2.28(\mathrm{~s}, 3 \mathrm{H}) ;{ }^{13} \mathrm{C}$ NMR (100 MHz, $\left.\mathrm{CDCl}_{3}\right) \delta 153.8,147.3,145.6,139.5,136.6,134.5$, 132.8, 131.0, 130.4, 130.2, 128.9, 128.7, 126.1, 120.5, 21.3, 21.1. HRMS (ESI) $m / z$ calcd for $\mathrm{C}_{23} \mathrm{H}_{19}{ }^{35} \mathrm{ClN}_{3} \mathrm{O}_{2} \mathrm{~S}[\mathrm{M}+\mathrm{H}]^{+}: 436.0887$, found 436.0885; HRMS (ESI) $\mathrm{m} / z$ calcd for $\mathrm{C}_{23} \mathrm{H}_{19}{ }^{37} \mathrm{ClN}_{3} \mathrm{O}_{2} \mathrm{~S}$ [M + $\mathrm{H}]^{+}: 438.0857$, found 438.0859 .

3n: (red crystal, $46 \%) .{ }^{1} \mathrm{H}$ NMR $\left(400 \mathrm{MHz}, \mathrm{CDCl}_{3}\right) \delta 7.87-7.68$ (m, 4H), 7.57-7.42 (m, 2H), 7.29-7.22 (m, 1H), 7.20-7.13 (m, 2H), 7.09 (d, $J=8.4 \mathrm{~Hz}, 2 \mathrm{H}), 7.04-6.98(\mathrm{~m}, 2 \mathrm{H}), 6.94-6.85(\mathrm{~m}$,
2H), $2.33(\mathrm{~s}, 3 \mathrm{H}), 2.17(\mathrm{~s}, 3 \mathrm{H}) ;{ }^{13} \mathrm{C}$ NMR $\left(100 \mathrm{MHz}, \mathrm{CDCl}_{3}\right)$ $\delta$ 154.2, 147.5, 147.0, 139.2, 134.4, 133.6, 133.0, 132.5, 130.4, $130.2,130.1,128.8,128.6,128.3,128.0,127.8,126.8,125.8$, 125.0, 120.6, 21.3, 21.1. HRMS (ESI) $\mathrm{m} / \mathrm{z}$ calcd for $\mathrm{C}_{27} \mathrm{H}_{22} \mathrm{~N}_{3} \mathrm{O}_{2} \mathrm{~S}$ $[\mathrm{M}+\mathrm{H}]^{+}:$: 452.1433, found 452.1435.

\section{Conflicts of interest}

There are no conflicts to declare.

\section{Acknowledgements}

The authors gratefully acknowledge financial support by funding from the Natural Science Foundation of China (21572197 and U1702286) and Nanyang Technological University (RG120/ 18).

\section{Notes and references}

1 (a) Y. Wang, W.-X. Zhang, Z. Wang and Z. Xi, Angew. Chem., Int. Ed., 2011, 50, 8122-8126; (b) G. Meng, M. Zheng, M. Dong, M. Wang, A. Zheng and Z. Guo, J. Heterocycl. Chem., 2016, 53, 588-594; (c) G. S. Kumar, A. S. Kumar and H. M. Meshram, Synlett, 2016, 399-403.

2 (a) J. P. Brincat, E. Carosati, S. Sabatini, G. Manfroni, A. Fravolini, J. L. Raygada, D. Patel, G. W. Kaatz and G. Cruciani, J. Med. Chem., 2011, 54, 354-365; (b) S. Bae, H.-G. Hahn, K. D. Nam and H. Mah, J. Comb. Chem., 2005, 7, 7-9.

3 (a) K. D. Hargrave, F. K. Hess and J. T. Oliver, J. Med. Chem., 1983, 26, 1158-1163; (b) W. C. Patt, H. W. Hamilton, M. D. Taylor, M. J. Ryan, D. G. Taylor, C. J. C. Connolly, A. M. Doherty, S. R. Klutchko and I. Sircar, J. Med. Chem., 1992, 35, 2562-2572; (c) F. Haviv, J. D. Ratajczyk, R. W. DeNet, F. A. Kerdesky, R. L. Walters, S. P. Schmidt, J. H. Holms, P. R. Young and G. W. Carter, J. Med. Chem., 1988, 31, 1719-1728; (d) K. Tsuji and H. Ishikawa, Bioorg. Med. Chem. Lett., 1994, 4, 1601-1606; (e) F. W. Bell, A. S. Cantrell, M. Hoegberg, S. R. Jaskunas, N. G. Johansson, C. L. Jordan, M. D. Kinnick, P. Lind and J. M. Morin, J. Med. Chem., 1995, 38, 4929-4936.

4 (a) N. Pietrancosta, A. Moumen, R. Dono, P. Lingor, V. Planchamp, F. Lamballe, M. Bähr, J.-L. Kraus and F. Maina, J. Med. Chem., 2006, 49, 3645-3652; (b) S. D. Barchéchath, R. I. Tawatao, M. Corr, D. A. Carson and H. B. Cottam, J. Med. Chem., 2005, 48, 6409-6422.

5 (a) A. Hantzsch and J. H. Weber, Chem. Ber., 1887, 20, 31183132; (b) S. Kasmi, J. Hamelin and H. Benhaoua, Tetrahedron Lett., 1998, 39, 8093-8096; (c) C. Bodhak and A. Pramanik, J. Org. Chem., 2019, 84, 7265-7278.

6 T. N. Birkinshaw, S. A. Harkin, P. T. Kaye, G. D. Meakins and A. K. Smith, J. Chem. Soc., Perkin Trans. 1, 1982, 939-943.

7 W. J. Middleton, J. Org. Chem., 1966, 31, 3731-3734.

8 J. Gasteiger and C. Herzig, Tetrahedron, 1981, 37, 2607-2611. 9 (a) N. De Kimpe, M. Boelens and J.-P. Declercq, Tetrahedron, 1993, 49, 3411-3424; (b) W. Zheng, A. Degterev, E. Hsu, 
J. Yuan and C. Yuan, Bioorg. Med. Chem. Lett., 2008, 18, 49324935.

10 (a) J. Svetlik, F. Turecek and I. Goljer, J. Org. Chem., 1990, 55, 4740-4744; (b) J. Liebscher and E. Mitzner, Tetrahedron Lett., 1985, 26, 1835-1838.

11 F. Tittelbach, S. Vieth and M. Schneider, Eur. J. Org. Chem., 1998, 515-520.

12 M. D'Hooghe, A. Waterinckx and N. De Kimpe, J. Org. Chem., 2005, 70, 227-232.

13 Y. Sanemitsu, S. Kawamura, J. Satoh, T. Katayama and S. Hashimoto, J. Pestic. Sci., 2006, 31, 305-310.

14 E. Schmitz and H. Striegler, J. Prakt. Chem., 1970, 312, 359365.

15 (a) H. Beyer, W. lässig and E. Bulka, Chem. Ber., 1954, 87, 1385-1392; (b) H. Beyer and G. Wolter, Chem. Ber., 1956, 89, 1652-1658.

16 (a) A. Bijev and P. Prodanova, Synth. Commun., 2006, 36, 3095-3101; (b) X. C. Wang, F. Wang, Z. J. Quan, Z. Zhang and M. G. Wang, J. Heterocycl. Chem., 2006, 43, 1473-1477; (c) A. Manaka, T. Ishii, K. Takahashi and M. Sato, Tetrahedron Lett., 2005, 46, 419-422; (d) J. Schmeyers and G. Kaupp, Tetrahedron, 2002, 58, 7241-7250.
17 (a) S. Murru, C. B. Singh, V. Kavala and B. K. Patel, Tetrahedron, 2008, 64, 1931-1942; (b) C. B. Singh, S. Murru, V. Kavala and B. K. Patel, Org. Lett., 2006, 8, 5397-5399; (c) C.-Y. Chen, I. J. Barve and C.-M. Sun, ACS Comb. Sci., 2016, 18, 638-643.

18 (a) V. Mane, S. T. Sivanandan, R. G. Santana, A. Beatriz, E. N. da Silva Júnior and I. N. N. Namboothiri, J. Org. Chem., 2020, 85, 8825-8843; (b) J.-W. Zhang, L.-S.-H. Yu, J.-L. Dong, Q.-C. Sun and J.-W. Xie, Synlett, 2018, 603-608; (c) J. Feng, X. Yuan, W. Luo, L. Lin, X. Liu and X. Feng, Chem.-Eur. J., 2016, 22, 15650-15653; (d) V. Kumar, A. Awasthi, A. Metya and T. Khan, J. Org. Chem., 2019, 84, 11581-11595; (e) W. E. Parham and J. L. Bleasdale, J. Am. Chem. Soc., 1951, 73, 4664-4666; (f) J.-W. Xie, Z. Wang, W.-J. Yang, L.-C. Kong and D.-C. Xu, Org. Biomol. Chem., 2009, 7, 4352-4354; (g) S. E. Denmark, V. Guagnano, J. A. Dixon and A. Stolle, J. Org. Chem., 1997, 62, 4610-4628. 19 T. Okino, Y. Hoashi, T. Furukawa, X. Xu and Y. Takemoto, J. Am. Chem. Soc., 2005, 127, 119-125.

20 A. C. Dawsey, V. Li, K. C. Hamilton, J. Wang and T. J. Williams, Dalton Trans., 2012, 41, 7994-8002. 